\title{
Effects of Aqueous Extracts of Moringa Oleifera on Lipid Profile of Tamoxifen-Induced Atherosclerotic Rats
}

\author{
Abeer Atef Shalan ${ }^{1}$ El Said EI Sherbini El Said ${ }^{2}$, Mahmoud Gamil ElSebaei ${ }^{2}$ and Reda \\ Eldemerdash $^{3}$
}

\author{
${ }^{1}$ Directorate of Veterinary Medicine \\ ${ }^{2}$ Biochemistry Department,Faculty of Veterinary Medicine, Mansoura University, Mansoura, Egypt. \\ ${ }^{3}$ Urology and Nephrology Center, Mansoura University, Mansoura, Egypt \\ dr.abeershalan@yahoo.com,sshrbini@mans.edu.eg,mahmoudgmail30@yahoo.com, \\ reda_eldemerdash@yahoo.com
}

\begin{abstract}
Moringa oleifera (M. oleifera) is, native of the Indian subcontinent, plant of angiosperm utilized in food and medicine. It is cultivated in all tropical and sub-tropical regions of the world. The therapeutic prophylactic and nutritional uses of this plant are extensively used. Moringa oleifera is an edible plant. A wide variety of nutritional and medicinal virtues have been attributed to its roots, bark, leaves, flowers, fruits, and seed. Phytochemical analyses have shown that its leaves are particularly rich in potassium, calcium, phosphorous, iron, vitamins $A$ and $D$, essential amino acids, as well as such known antioxidants such as $\beta$ carotene, vitamin $C$, and flavonoids. Various therapeutic methods used in treatment of atherosclerosis but fail to prevent complication of cardiovascular origin, so we used medicinal plant in therapy for excluding complication.Moringa oleifera is used as hypocholesterolaemic effect in Sprague dawley rats as shown in recent studies.However, we need more data about scientific uses of this natural product.The aim of the present study was to evaluate the effect of aqueous extract of moringa oleifera leaves on Lipid profile; triglycerides (TGs), cholesterol, high density lipoproteins (HDL) and low density lipoproteins (LDL) and very low density lipoprotein (VLDL), as well as on liver tissues for superoxide dismutase (SOD), catalase (CAT), malondialdehyde (MDA),Total antioxidant capacity (TAC), and gene expression for LDLgene.The extract was administered at two doses, $200 \mathrm{mg} / \mathrm{kg}$ bw and 300 $\mathrm{mg} / \mathrm{kg}$ bw respectively orally for a period of 30days. Tamoxifen with a dose of $0.5 \mathrm{mg} / \mathrm{kg}$ b.wt.by $\mathrm{S} / \mathrm{C}$ for 5 consequtive days induced atherosclerotic rats showed hyperlipidemia ,hypercholesterolemia,loweing in TAC,SOD, and MDA, while increase the catalase ratio.Moringa groups showed cure most of the deviation near to the normal ratio. The present study reveals that moringa oleifera extract possesses antiatherosclerotic and antihyperlipedemic effect in atherosclerotic induced rats by tamoxifen .
\end{abstract}

Keywords: Hypercholesterolemia; Atherosclerotic events; Hypocholesterolemic agent; Moringa oleifera; Tamoxifen.

\section{INTRODUCTION}

The objective of the present work was to evaluate the effect of Moringa oleifera on atherosclerosis in rat. Atherosclerosis is a disease of blood vessels known as hardening of the arteries. It is characterized by the accumulation of fatty substance, cholesterol, cellular waste products, calcium and other substances in the inner lining of an artery. Major complications of atherosclerosis include angina pectoris, myocardial infarction and stroke, which are recognizedas leading causes of morbidity and mortality] 16]Medicinal plants have a gold future due to about half million plants all over the world, and most of them not investigate yet as a medical activities So encourage researchers to work for clarify the active ingredients extracted from medicinal plants... Moringa oleifera belongs to the single genus monogeneric family Moringaceae and is well distributed in Africa and Asia.It consider a good source of vitamins and amino acids, it has medical uses,] 16]. It has been used in the treatment of numerous disease conditions, [15].including heart disease and obesity due to its hypocholesterolemic property] 5]. The major carrier of cholesterol in plasma is LDL and involved in the process of atherosclerosis. LDL was originally thought to be monodisperse, i.e. composed of a distribution of particles differing slightly in size from one another; the mean particle size was about $250 \mathrm{~A}^{\circ}$ in diameter and the peak density was $1.035 \mathrm{~g} / \mathrm{ml}$. It was noted first in patients with hypertriglyceridaemia that fractions of LDL with differing size and density existed, and the seminal work of] 8]. Obesity has emerged as a major health problem and risk factor for various disorders 
worldwide,[ 17].obesity are defined as abnormal or excessive fat accumulation triggered by disproportion in energy intake and expenditure,[14].Tamoxifen, a non-steroidal anti-oestrogen, used in the treatment of breast cancer, The anti-oestrogen drug exerts its effect by binding to oestrogen receptors of the tumor cells,[10]. Treatment with tamoxifen is associated with an increased risk of fatty liver, and treatment with tamoxifen develops $43 \%$ of women with breast cancer. The first 2 years of treatment with tamoxifen may develop steatosis,[12]. Few literatures concerning aqueous extract of Moringa oleifera, so that, the role of aqueous extract of Moringa oleifera as a reference against tamoxifen induced atherosclerosis is evaluated in this present study.

\section{MATERial AND MeTHODS}

\subsection{Animals}

Fifty male rats of Sprague Dawley strain weighing (250 - 300) gm were obtained from Nile Center of Experimental Research, Mansoura, Egypt. The rats were maintained under standard laboratory conditions in an air conditioned room and housed in stainless steel cages one per cage at temperature $22 \pm 3{ }^{\circ} \mathrm{C}$ and relative humidity $30-70 \%$. The animal diet was given ad libitum. Animals were acclimation for one week prior to the experimental work. All animals received human care in compliance with the guidelines of the Animal Care and Use Committee of Mansoura University.

\subsection{Tamoxifen}

Tamoxifen was purchased from local pharmacy and dissolved at concentration of $1 \mathrm{mg} / \mathrm{mL}$ in sesame oil containing $1 \%$ benzyl alcohol from local apothecary, Mansoura, Egypt.

\subsection{Preparation of Plant}

The leaves of Moringa oleifera were authenticated by local market, Mansoura, Egypt. The extraction method was performed as described by (Ghasi, et. al, 2000) (5).

\subsection{Chemicals}

Lipid profile; Total Lipid (ABC Diagnostic) triglycerides (TGs), cholesterol, high density lipoproteins (HDL) and low density lipoproteins (LDL) and very low density lipoprotein (VLDL) (Spinreact)

Antioxidant, superoxide dismutase (SOD), catalase (CAT), malondialdehyde (MDA), Total antioxidant capacity (TAC) (BIODIAGNOSTC)

\subsection{Experimental Protocol}

Fifty adult male rats were allocated to one of five groups subjected to the following treatments:

Group (1): 10 rats were served as a negative control group.

Group (2): 10 rats were given TMX at a dose of $0.5 \mathrm{mg} / \mathrm{kg}$ b.wt.by S/C for 5 consequtive days as a positive control group.

Group (3): 10 rats were were given TMX at a dose of $0.5 \mathrm{mg} / \mathrm{kg}$ b.wt.by S/C and were given water extract of Moringa oleifera leaves at a dose $(200 \mathrm{mg} / \mathrm{kg}$ bw) orally by stomach tube for 30 days.

Group (4): 10 rats were given TMX at a dose $0.5 \mathrm{mg} / \mathrm{kg}$ b.wt.by S/C and were given water extract of Moringa oleifera leaves at a dose $(300 \mathrm{mg} / \mathrm{kg}$ bw) orally by stomach tube for 30 days.

Group (5): 10 rats were given water extract of Moringa oleifera leaves at a dose (200mg/kg bw) orally by stomach tube for 30days.

At the end of the experimental period, the rats of each treated group were fasted overnight, and sacrificed under slight halothan anaesthesia. Blood was collected by cardiac puncture. Serum were separated by centrifugation at $860 \mathrm{xg}$ for $20 \mathrm{~min}$. and determination of triglycerides (TGs), cholesterol, high density lipoproteins (HDL) and low density lipoproteins (LDL) and very low density lipoprotein (VLDL), were carried out using enzymatic colorimetric kits according to [18].and liver tissues for superoxide dismutase (SOD), catalase (CAT) [2], Malondialdehyde (MDA),[13], Total antioxidant capacity (TAC) [7], Another part of liver was washed in $0.9 \%$ saline, minced and homogenized, for determination of gene expression for low density lipoproteins gene ( LDL gene) using semiquantitative (RT-PCR ARKTIK, thermal cycler, USA). 


\subsection{Gene Expression of LDL by Real-Time PCR}

To determine the effects of aqueous extract of Moringa and Tamoxifen drug on hepatic cytochrome LDL at gene level by relative expression. Glyceraldehyde-3-phosphate dehydrogenase was included as an internal control. Briefly, Total RNA was extracted from treated liver rat, as well as from untreated and sclerotic liver rats by SV total RNA isolation system according to the manufacturer's instructions (Promega Corporation, Madison, WI, USA). Reverse transcription was done using $1 \mu \mathrm{g}$ total RNA and a cDNA kit (RevertAid First Strand cDNA, Thermo Scientific, Lithuania). Two $\mu \mathrm{L}$ of the cDNA sample was mixed with 10 pmol of each primer and $10 \mu \mathrm{L}$ of Sybr green master mix. (SensiFast SYBR, BIOLINE, UK). Distilled water was added to a volume of $20 \mu \mathrm{L}$, and the resulting mixture was subjected to real-time PCR amplification. The cycling parameters were as follows: initial denaturation at $95{ }^{\circ} \mathrm{C}$ for 2 minutes, followed by 40 cycles of $94^{\circ} \mathrm{C}$ for 15 seconds, annealing and extension at $60{ }^{\circ} \mathrm{C}$ for 1 minute and the final melting curve was done to show the specificity of primers. The specific oligonucleotide primers were shown in Table 1. The calculation for relative gene expression was done according to] 9].

Table1. The primer sequences used for real time PCR assay in rats

\begin{tabular}{|l|l|l|l|}
\hline Gene & \multicolumn{1}{|c|}{ Sequences (5'-3') } & Accession No. & \multicolumn{1}{c|}{$\begin{array}{c}\text { PCR } \\
\text { Products }\end{array}$} \\
\hline $\begin{array}{l}\text { LDL } \\
\text { Low Density Lipoprotein }\end{array}$ & $\begin{array}{l}\text { F: AGCATAAACTTTGACAACCCAGTCT } \\
\text { R: TCCTGGCTGCGGCAAAT }\end{array}$ & NM_175762.2 & 71 bp \\
\hline $\begin{array}{l}\text { GAPDH } \\
\text { Glyceraldehyde-3-phosphate } \\
\text { dehydrogenase }\end{array}$ & $\begin{array}{l}\text { F: CCAGGGCTGCCTTCTCTTGT } \\
\text { R: CTGTGCCGTTGAACTTGCCG }\end{array}$ & NM_017008.4 & 123 bp \\
\hline
\end{tabular}

\subsection{Histopathological Examination}

The liver tissues, fixed in 10\% formalin, was dehydrated through the ascending series of ethyl alcohol (50-100), cleared in xylene, infiltrated and embedded in paraffin wax. Transverse sections of liver were cut at thickness of $5 \mu \mathrm{m}$ and stained with Mayer's Haematoxylin and Eosin (H\&E) stains according to [19].

\subsection{Statistical Analysis}

The results were reported as mean $\pm \mathrm{SEM}^{*}$. The statistical analysis was carried out using ShapiroWilk test for assessment of the normality of data, one way ANOVA* with LSD* Post Hoc test and the Pearson correlation coefficient. Values were considered significant at $\mathrm{P}<0.05$. The software SPSS, version 19 was used.

*SEM (Standard error of mean), ANOVA (analysis of variance), LSD (Least significant Difference).

\section{RESUlts}

The results of lipid profile demonstrated that the rats treated with tamoxifen caused significant $(\mathrm{P} \leq 0.05)$ peroxidative damage as evidenced by liver enzymes and antioxidant defense system (table 2, $3)$.

Table2. Serum total lipids, cholesterol, and triglycerides activity in control and different rat groups

\begin{tabular}{|l|l|l|l|}
\hline \multicolumn{1}{|c|}{ Group } & Total lipids & cholesterol & Triglycerides \\
\hline Control & $670 \pm 19.37^{\mathrm{c}}$ & $68.3 \pm 3.53^{\mathrm{c}}$ & $86.4 \pm 8.6^{\mathrm{c}}$ \\
\hline Tamoxafen & $1044 \pm 204^{\text {abde }}$ & $87.3 \pm 1.62^{\text {abde }}$ & $116 \pm 14.04^{\text {abde }}$ \\
\hline Moringa(200mg) + Tamoxafen & $518 \pm 30.38^{\mathrm{c}}$ & $61.7 \pm 1.61^{\mathrm{c}}$ & $74.3 \pm 3.89^{\mathrm{c}}$ \\
\hline Moringa(300mg) + Tamoxafen & $500 \pm 41.72^{\mathrm{c}}$ & $65.01 \pm 4.43^{\mathrm{c}}$ & $73.6 \pm 4.89^{\mathrm{c}}$ \\
\hline Moringa(200mg) & $640 \pm 27.29^{\mathrm{c}}$ & $60.7 \pm 2.14^{\mathrm{c}}$ & $79.4 \pm 4.02^{\mathrm{c}}$ \\
\hline
\end{tabular}

Data expressed as mean \pm SEM. ${ }^{a}$ significant $v$ s control, ${ }^{b}$ significant vs Moringa, ${ }^{c}$ significant vs Tamoxafen, ${ }^{d}$ significant vs (200mg) Moringa+Tamoxafen, ${ }^{e}$ significant vs (300mg) Moringa+Tamoxafen.

- All P values of significant statistics were $<0.001$ except for the followings:

Triglycerides (control vs Tamoxafen < 0.05), (moringa vs Tamoxafen < 0.01), (Tamoxafen vs 1 Moringa+Tamoxafen <0.01), (Tamoxafen vs 1.5 Moringa+Tamoxafen < 0.01) 
Abeer Atef Shalan et al.

Table3. Serum high density lipoproteins (HDL), low density lipoproteins (LDL) and very low density lipoproteins (VLDL)

\begin{tabular}{|l|l|l|l|}
\hline Group & HDL & LDL & VLDL \\
\hline Control & $33.4 \pm 4.96^{\mathrm{c}}$ & $17.6 \pm 4.58^{\mathrm{b}}$ & $17.3 \pm 1.72^{\mathrm{c}}$ \\
\hline Tamoxafen & $53.6 \pm 2.24^{\text {abde }}$ & $10.5 \pm 5.23$ & $23.2 \pm 2.81^{\text {abde }}$ \\
\hline Moringa (200mg) + Tamoxafen & $38.2 \pm 2.36^{\mathrm{c}}$ & $8.7 \pm 1.61$ & $14.9 \pm 0.78^{\mathrm{c}}$ \\
\hline Moringa (300mg) + Tamoxafen & $36.01 \pm 1.89^{\mathrm{c}}$ & $14.29 \pm 2.93$ & $14.7 \pm 0.97^{\mathrm{c}}$ \\
\hline Moringa(200mg) & $37.3 \pm 1.07^{\mathrm{c}}$ & $7.47 \pm 1.26^{\mathrm{a}}$ & $15.78 \pm 0.75^{\mathrm{c}}$ \\
\hline
\end{tabular}

Data expressed as mean \pm SEM. ${ }^{a}$ significant $v$ s control, ${ }^{b}$ significant $v$ s Moringa, ${ }^{c}$ significant vs Tamoxafen, ${ }^{d}$ significant vs Moringa(200mg) + Tamoxafen, ${ }^{e}$ significant vs Moringa(300mg) +Tamoxafen.

- All P values of significant statistics were <0.01 except for the followings:

i) HDL (control vs Tamoxifen <0.001).

ii) LDL (control vs Moringa <0.05).

iii) 3-VLDL (control vs Tamoxifen <0.05).

Table4. Liver tissues for Total antioxidant capacity (TAC), superoxide dismutase (SOD), Malondialdehyde (MDA) and catalase (CAT)

\begin{tabular}{|l|l|l|l|l|}
\hline Group & TAC & SOD & MDA & Catalase \\
\hline Control & $0.76 \pm 0.02^{\mathrm{c}}$ & $80.7 \pm 4.18^{\text {bde }}$ & $3.3 \pm 0.19^{\text {bde }}$ & $501 \pm 14.97^{\text {bcde }}$ \\
\hline Tamoxafen & $0.7 \pm 0.02^{\text {ad }}$ & $69.3 \pm 6.39^{\text {bde }}$ & $6.9 \pm 0.26^{\text {abde }}$ & $706 \pm 21.3^{\text {abde }}$ \\
\hline Moringa (200mg) + Tamoxafen & $0.77 \pm 0.01^{\text {bc }}$ & $162 \pm 10.96^{\text {abc }}$ & $2.8 \pm 0.12 \mathrm{ce}$ & $602 \pm 6.34^{\text {abce }}$ \\
\hline Moringa (300mg) + Tamoxafen & $0.75 \pm 0.02^{\text {abcd }}$ & $165 \pm 11.5^{\text {abc }}$ & $3.6 \pm 0.1 \mathrm{~cd}$ & $651 \pm 12.88^{\text {abcd }}$ \\
\hline Moringa(200mg) & $0.73 \pm 0.02^{\text {d }}$ & $196 \pm 3.72^{\text {acde }}$ & $3.4 \pm 0.23 \mathrm{c}$ & $443 \pm 19.23^{\text {acde }}$ \\
\hline
\end{tabular}

Data expressed as mean \pm SEM. ${ }^{a}$ significant $v$ s control, ${ }^{b}$ significant vs Moringa, ${ }^{c}$ significant vs Tamoxafen, ${ }^{d}$ significant vs Moringa(200mg) +Tamoxafen, ${ }^{e}$ significant vs Moringa(300mg) +Tamoxafen .

- All P values of significant statistics were <0.001 except for the followings:

i) TAC (Control vs tamoxafen <0.05), (moringa vs moringa (200mg) +tamoxafen <0.05), (Tamoxafen vs moringa $(200 m g)+$ tamoxafen $<0.05)$.

ii) SOD (moringa vs 1 moringa+tamoxafen <0.01), (moringa vs 1.5 moringa(300mg) + tamoxafen $<0.01)$.

iii) MDA (control vs moringa <0.05), (control vs moringa(200mg) + tamoxafen <0.05), (moringa vs tamoxafen <0.01), (moringa vs moringa(300mg) + tamoxafen <0.01), (Tamoxafen vs moringa(200mg)+tamoxafen <0.01), moringa(200mg) +tamoxafen vs moringa(300mg) + tamoxafen $<0.05)$.

iv) Catalase (Control vs moringa < 0.01), (Tamoxafen vs moringa(300mg) +tamoxafen <0.05), moringa(200mg) +tamoxafen vs moringa(300mg) + tamoxafen <0.05).

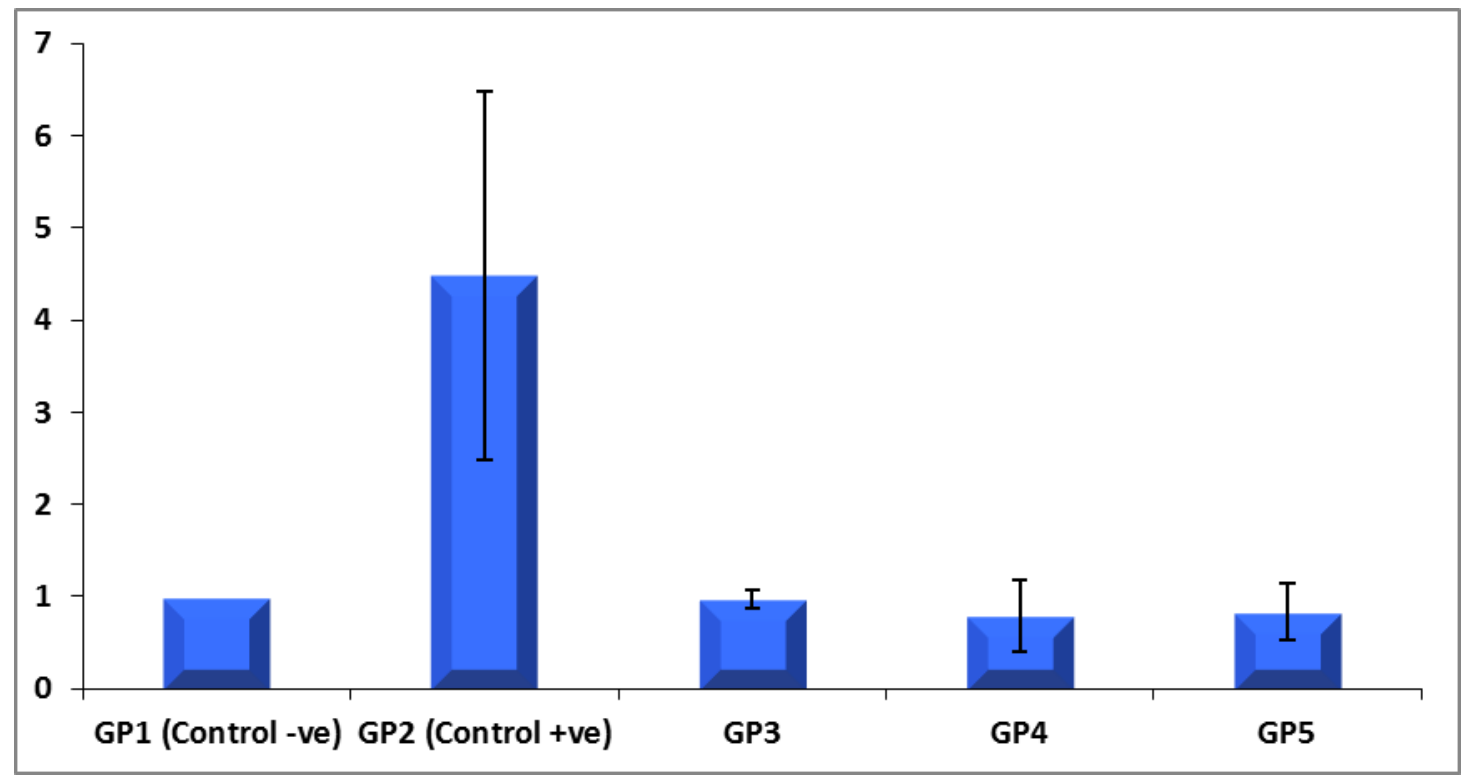

Fig1. Relative expression of LDL gene in untreated and treated rats groups 


\section{LDL GENE EXPRESSION}

To determine the effects of aqueous extract of Moringa and Tamoxifen drug on hepatic cytochrome LDL at gene level, relative quantitation RT-PCR was determined(6). The mean and standard deviation for each group were calculated as shown in fig.1. Relative gene expression depends on the expression of mRNA for LDL gene relative to that of sham group (negative control). The expression of this gene was 4.8 folds in the case of TMX for 5 days (GP2) more than negative control. On otherwise the groups 3, 4 and 5 were changed to 0.97 and 0.799 relative expressions, respectively. The changing of mRNA relative expression for LDL gene was affected by the Moringa oleifera oral treatment. Also, in the case of Moringa oleifera only without atherosclerosis the relative expression of LDL was less than negative control (0.83).
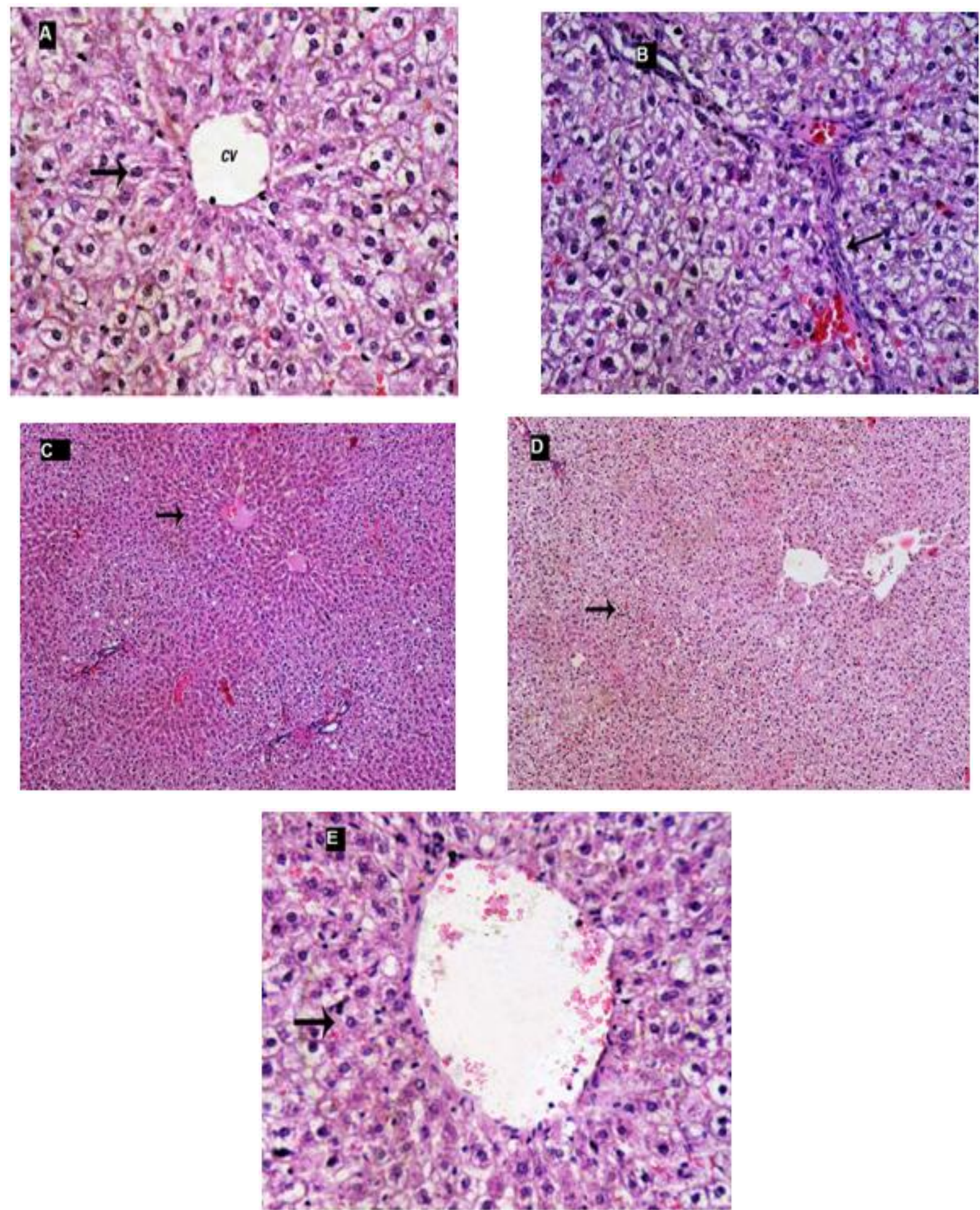
A Histopathological study of liver sections of normal control (G1) rats revealed normal hepatocytes, normal liver architecture as shown in Fig. A, Liver of Tamoxifen treated rats (G2) Fig. B, liver showing degenerative change of hepatocytes, congestion of hepatic sinusoids and marked fibroblastic proliferation forming fibrous septa (arrow) (HE, 400x). Examination of liver sections of atherosclerotic rats treated with the aqueous extract of moringa oleifera (G3)in a daily dose $(200 \mathrm{mg})$ for 30 day decrease the severity of histopathological changes, liver showing normal architecture and normal hepatocytes (arrow), (HE,100x) as shown in fig.C, The liver of atherosclerotic rats treated with the aqueous extract of Moringa oleifera (G4) in a daily dose $(300 \mathrm{mg})$ for 30 day showed normal architecture with degenerative change of hepatocytes (arrow) and congestion of hepatic sinusoids. (HE, 100x) Fig.D, Histopathological examination of livers of normal rats treated with $1 \mathrm{ml}$ moringa daily for 30 days (G5) revealed normal histological architecture with normal hepatocytes (arrow). (HE, 100x), Fig. E.

\section{DiSCUSSION}

The model of inducing atherosclerosis in animal is the administration of tamoxifen as described by] 3].Several studies demonstrated the increased levels of total lipids, cholesterol, and triglycerides and lowering in antioxidants into blood stream after administration of Tamoxifen. In the present study tamoxifen administration caused elevation of total lipids,cholesterol , triglycerides, HDL, VLDL and catalase, while cause decrease in TAC,SOD, and MDA. Histopathological investigation reveal degenerative change of hepatocytes, congestion of hepatic sinusoids and marked fibroblastic proliferation forming fibrous septa, where agreement with] 1].These changes may be due to low level of antioxidants in liver tissues. In folk medicine, herbal extracts are often used to prevent heart diseases and improve lipid profile. M. oleifera leaves extract in our study showed lowering the cholesterol, triglycerides and VLDL, and this agreement with] 6]. M. oleifera in a dose of (200mg) much lowering lipid profile more than M. oleifera $(300 \mathrm{mg})$ especially cholesterol and LDL. However, the treated rats with moringa showed marked decrease in degeneration and fibrosis in liver tissues and this may be due to increasing antioxidant enzymes inside liver cells.

\section{Conclusion}

Administration of tamoxifen to the rats lead to atherosclerosis .Therapeutic effect of Moringa oleifera on atherosclerotic rats, through protecting tissues from higher levels of cholesterol, triglycerides and total lipids , also increasing the antioxidant enzymes. LDL gene was affected by the Moringa oleifera oral treatment. Also, in the case of Moringa oleifera only without atherosclerosis the relative expression of LDL was less than negative control.

\section{CONFLICT OF INTEREST}

The authors declare no financial or commercial conflict of interest.

\section{ACKNOWLEDGEMENT}

Authors would thank to Nile Center of Experimental Research, Mansoura, Egypt for their technical and financial support.

\section{REFERENCES}

[1] Aattar AA... Comparative Physiological Study on the Effect of Rosemary, Tarragon and Bay Leaves Extract on Serum Lipid Profile of Quail, Coturnix coturnix. Saudi J Biol Sci.; 13:1- 98. (2006)

[2] Aebi H., Catalase: In: Methods of enzymatic analysis analysis, Bergemeyer, H V (ed), Academic Press, New York, NY, USA. pp: 673-684 (1974).

[3] Christopher J. Lelliott,1 Miguel Lo'pez,1 R. Keira Curtis, Nadeene Parker,Matthias Laudes, Giles Yeo,Mercedes Jimenez-Liñan, Johannes Grosse, Asish K. Saha, David Wiggins, David Hauton, Martin D. Brand, Stephen O'Rahilly, Julian L. Griffin, Geoffrey F. Gibbons, and Antonio Vidal-Puig,2.. Transcript and metabolite analysis of the effects of tamoxifen in rat liver reveals inhibition of fatty acid synthesis in the presence of hepatic steatosis. FASEB Journal.19.11089-1119.( 2005)

[4] Francis G, Makkar H P S and Becker K 2005 Products from little researched plants as aquaculture feed ingredients. Retrieved February 24, (2005). 
[5] Ghasi S., Nwobodo E., Ofili J. O... Hypocholesterolemic effects of crude extract of leaf of Moringa oleifera Lam in high-fat diet fed Wistar rats. J. Ethnopharmacol. 69, 212510.1016/S0378-8741(99)00106-3. (2000)

[6] Glugliano, D., Ceriello, A. and Paolisso, G... Oxidative stress and diabetic vascular complications. Diabet. Care. 19: 257-267. (1996)

[7] Koracevic D., K+oracevic G., Djordjevic V., Method for the measurement of antioxidant activity in human fluids, J ClinPathol. 54,356-61 (2001).

[8] Krauss, R. and Burke D... J. Lipid Res. 23, 97-104 ( 1982)

[9] Matthew T, Matthew Z, Taji S A and Zachariah S A review of Viricidal Ayurvedic Herbs of India for Poultry Diseases. Journal of American Holistic Veterinary Medicine Association 20(1): $17-20 .(2001)$

[10] Nicholson, R. I. And Walker, K. J. Use of LH-RH agonists in the treatment of breast disease. Proceedings of the Royal Society of Edinburgh. Section B. Biological Sciences, 95, pp 271-281. Doi: 10.1017/S0269727000010745. (1989).

[11] Nishikimi M., Appaji N., Yagi K., The occurrence of superoxide anion in the reaction of reduced phenazine-methosulfate and molecular oxygen,BiochemBiophys Res Commun. Jan 3146(2), 84-54 (1972).

[12] Nishino, M., K. Hayakawa, Y. Nakamura, T. Morimoto, and S. Mukaihara. Effects of tamoxifen on hepatic fat content and the development of hepatic steatosis in patients with breast cancer: high frequency of involvement and rapid reversal after completion of tamoxifen therapy. AJR Am. J. Roentgenol. 180: 129-134. .(2003)

[13] Ohkawa H., Ohishi N., Yagi K., Assay for lipid peroxides in animal tissues by thiobarbituric acid reaction, Analytical Biochemistry. 95, 351-358(1979).

[14] Panico,S. and Iannuzzi,A..Diatery fat composition and metabolic syndrome.Eur j lipid sci tech 106 (1):61-67.( 2004)

[15] Pfaffl, M. W. "A new mathematical model for relative quantification in real-time RT-PCR," Nucleic Acids Research, vol. 29, no. 9, article e45,.( 2001)

[16] Reinhardt, E. Health Watch: The Atlas of Heart Disease and Stroke. UNChronicle online, pp. 14. (2005)

[17] Roh C. and U. Jung,. "Screening of crude plant extracts with anti-obesity activity," International Journal of Molecular Sciences, vol. 13, no. 2, pp (2012)

[18] Tietz. N.W.Fundamental of clinical chemistry. $2^{\text {nd }}$ Edn.W.B.Saunders,Philadelphia, pp:878878.(1976).

[19] Weesner F.M., General zoological microtechniques. Scientific Book Agency Calcutta.P.86. (1968). 